\title{
Demonstration of the formation of porous silicon films with superior properties formed on polished (100) Si with screen-printed back contacts
}

\author{
Priyanka Singh $^{1}$, Shailesh N. Sharma ${ }^{1^{*}}$, G. Bhagavannarayana ${ }^{2}$, M. Husain $^{3}$ \\ and M. Lal ${ }^{1}$ \\ ${ }^{1}$ Electronic Materials Division, National Physical Laboratory, Dr. K.S. Krishnan Marg, \\ New Delhi-110 012, India. \\ ${ }^{2}$ Materials Characterization Division, National Physical Laboratory, Dr. K.S. Krishnan Marg, New \\ Delhi-110 012, India. \\ ${ }^{3}$ Department of Physics, Jamia Millia Islamia, New Delhi, India. \\ *email: shailesh@mail.nplindia.ernet.in
}

Keywords: porous silicon, back contacts, screen-printing \& thermal evaporation.

\begin{abstract}
Porous silicon (PS) layers were formed by anodization on polished substrates of $\left(\begin{array}{lll}1 & 0 & 0\end{array}\right)$ $\mathrm{Si}$ at different current densities for a fixed anodization time of 30 mins. using different screenprinted/evaporated back contacts (Ag, Al) respectively. The PS films has been characterized by high resolution X-ray diffraction (HRXRD), photoluminescence (PL), Scanning Electron Microscopy (SEM) and Fourier Transform Infrared (FTIR) techniques respectively. Porosity and thickness of PS layers were estimated by gravimetric analysis. The properties of PS formed using screen-printed Ag \& Al as the back contacts (SP-(Ag/Al)) was found to be superior as compared to the corresponding films with evaporated back contacts (EV-(Ag/Al)). The PS formed with screenprinted Ag \& Al-back contacts shows better crystalline perfection, higher stability, higher PL efficiency and negligible PL decay compared to that formed with evaporated Ag \& Al- as the back contact for the same current density and time of anodization.
\end{abstract}

\section{Introduction}

Screen-printed (SP) metallization is the most widely used contact formation technique for commercial Si-solar cells [1]. Although photolithography, metal evaporation or sputtering are the most well-established metallization techniques for solar cell fabrication [2]. However, these technologies are time consuming and expensive. Screen-printed contact technology is a more rapid metallization process and cost-effective as compared to sputtering and evaporation methods [1]. The utility of PS as an anti-refection coating in solar cells is well-known but it is imperative to prepare PS with less defect density in order to improve the solar cell efficiency [2]. In this work, by means of high resolution XRD, PL and FTIR studies, the superiority of PS films formed with Ag/Al as the screen-printed back contacts (SP-(Ag/Al)) have been demonstrated as compared to the corresponding ones with evaporated back contacts (EV-(Ag/Al)) for its possible application in photovoltaics.

\section{Experimental}

Boron doped $\mathrm{p}$ type Si polished wafers of (100) orientation, $1 \Omega-\mathrm{cm}$ resistivity and $285 \mu \mathrm{m}$ thickness with either (SP-(Ag/Al)) or (EV-(Ag/Al)) back contacts were used for preparing PS. PS was formed by the standard anodization process in a mixture of $\mathrm{HF}$ and $\mathrm{C}_{2} \mathrm{H}_{5} \mathrm{OH}$ (1:1 by volume) using $\mathrm{Si}$ as the anode and $\mathrm{Pt}$ as the counter electrode in an acid resistant container. The anodization was carried out at $\left(I_{d}\right) \sim 20$ to $60 \mathrm{~mA} \mathrm{~cm}{ }^{-2}$ for a fixed anodization time of 30 mins. The details of the contact formation \& characterization techniques can be found elsewhere [3]. 


\section{Results and Discussion}

The porosity and thickness of SP-(Ag/Al) \& EV(Ag/Al) PS films varied from 40-52\% and 100$200 \mathrm{~nm}$ respectively. Typical PL curves for SP(Ag/Al) \& EV-(Ag/Al) PS films formed at $\mathrm{I}_{d} \sim 20$ $\mathrm{mA} \mathrm{cm}$ are shown in Fig. 1. As evident from Fig.1, the absolute PL intensities $\left(\lambda_{\mathrm{PL} \text { Peak }} \sim 680\right.$ $690 \mathrm{~nm}$ ) of SP-(Ag/Al) PS films are quite high. However, for corresponding $\mathbf{E V}-(\mathbf{A g} / \mathbf{A l})$ films, a weak PL spectra $\left(\lambda_{\text {PL Peak }} \sim 740 \mathrm{~nm}\right)$ was obtained (Fig. 1). This difference in PL spectra for the same $I_{d} \sim 20 \mathrm{~mA} \mathrm{~cm}^{-2}$ could be apparently due to the fact that the silicon skeletons not being narrow enough for quantum confinement for $\mathbf{E V}-(\mathbf{A g} / \mathbf{A l})$ PS films. The stability of these PS films with different

back contacts were evaluated by monitoring the decay of the PL peak intensity as a function of

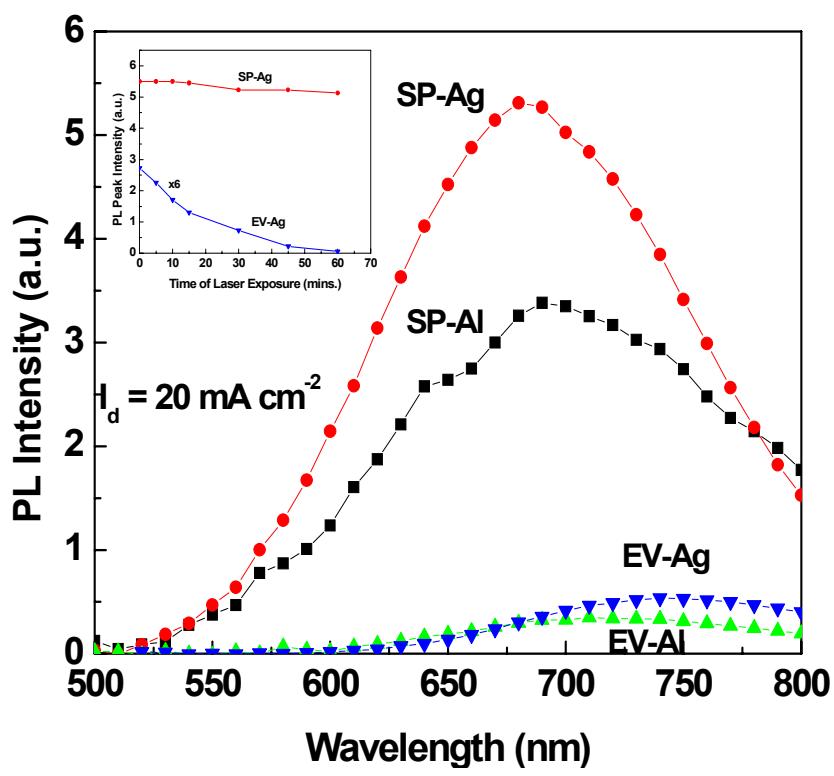

Fig. 1 Photoluminescence (PL) spectra of the (SP-(Ag/Al)) and (EV-(Ag/Al)) PS samples at $I_{d}=20 \mathrm{~mA} \mathrm{~cm}^{-2}$.

laser-exposure time. For PS film formed with Ag as the screen-printed back contact (SP-Ag), less PL decay was observed however, for PS film formed with Ag as the evaporated back contact (EV-Ag), a significant PL decay was observed as shown in the inset of Fig. 1. This is a good indication of the stability of the surface bond configurations for PS films with screen-printed back contacts.

Fig. 2 shows the FTIR spectra of SP-Ag and EV-Ag PS films. The Ag-SP PS films exhibits mainly Si-H related features at $\sim 2100 \mathrm{~cm}^{-1}$ (stretching mode), $907 \mathrm{~cm}^{-1}$ (bending mode) \& $660 \mathrm{~cm}^{-1}$ (wagging mode) and Si-O mode at $\sim 1045 \mathrm{~cm}^{-1}$ while $\mathbf{E V}-\mathbf{A g}$ films exhibits relatively less intense $\mathrm{Si}-\mathrm{H}$ features but prominent Si-O mode $\sim 1132 \mathrm{~cm}^{-1}$. Here, shift of Si-O related mode from $1042 \mathrm{~cm}^{-1}$ to $1132 \mathrm{~cm}^{-1}$ indicates an increase in the oxidation state $(\mathrm{x})$ of the $\mathrm{SiO}_{\mathrm{x}}$ species [3]. Thus, the effect of oxidation is felt more on $\mathbf{E V}-\mathbf{A g}$ PS films as compared to corresponding SP-Ag films. Similar results were also observed for SP-Al and EV-Al PS films as well. The inset of Fig. 2 shows the SEM images depicting the surface morphology of SP-Ag (right-inset) \& EV-Ag (left-inset) PS films for $\mathrm{I}_{\mathrm{d}} \sim 20 \mathrm{~mA}$ $\mathrm{cm}^{-2}$. Here, a plain featureless surface morphology similar to that of polished Si substrate is observed for EV-Ag film indicating little or no PS formation while for SP-Ag films, a distinct formation of PS indicating microstructural variations as observed by changes in SEM contrast is revealed.

Fig. 3 shows capacitance-voltage (C-V) curves measured between +2 and $-2 \mathrm{~V}$ at room temperature in the dark at $1 \mathrm{KHz}$ for $\mathbf{E V - A g}$ and SP-Ag PS films. The $\mathrm{C}-\mathrm{V}$ curves are similar to those of a typical MIS (metalinsulator-semiconductor) capacitor with a p-type substrate. From fig. 3, the $\mathrm{C}-\mathrm{V}$ curve corresponding to SP-Ag PS forms a proper hysteresis loop while $\mathbf{E V - A g}$

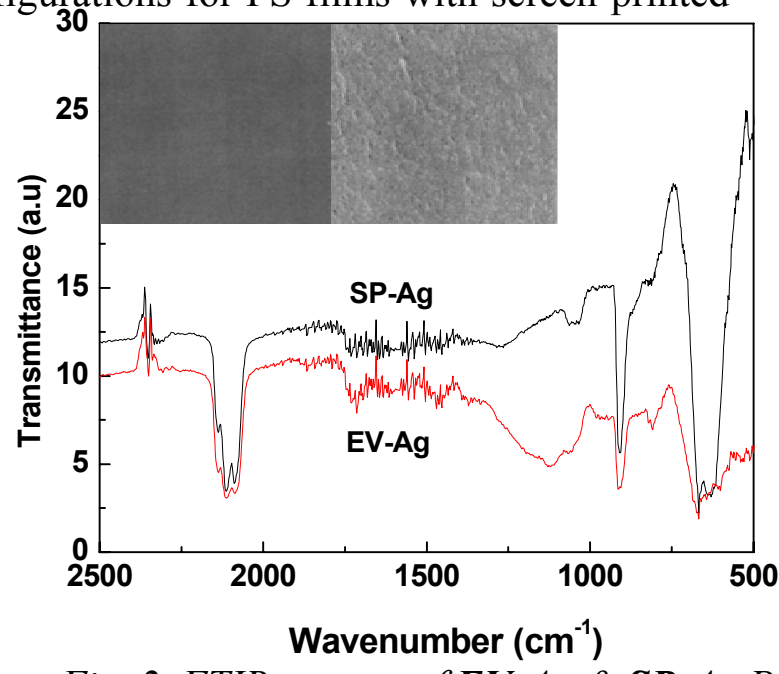

Fig. 2 FTIR spectra of $\boldsymbol{E} \boldsymbol{V}-\boldsymbol{A g} \& \boldsymbol{S P}-\boldsymbol{A g}$ PS films for $I_{d} \sim 20 \mathrm{~mA} \mathrm{~cm}^{-2}$. The left and right insets show their corresponding morphologies as depicted by SEM.

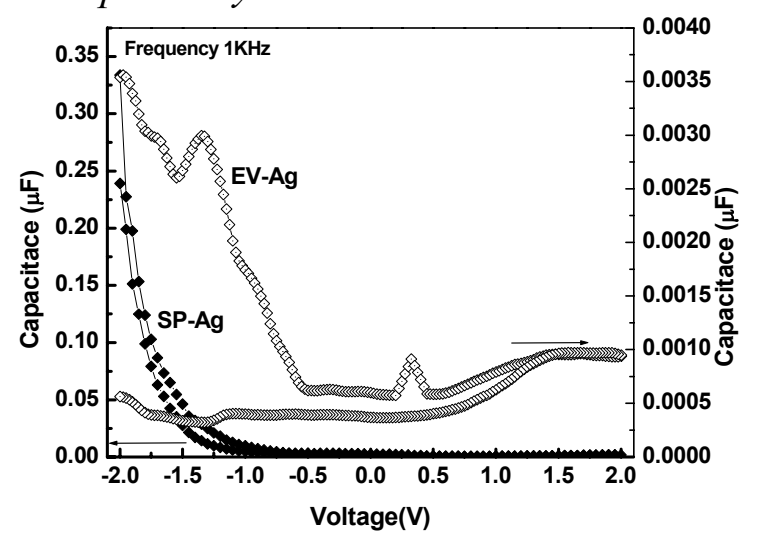

Fig. 3 Capacitance-Voltage (CV) characteristics for $\boldsymbol{E} \boldsymbol{V}-\boldsymbol{A g}$ and $\boldsymbol{S P}-\boldsymbol{A g}$ $P S$ films prepared at $I_{d}=20 \mathrm{~mA} \mathrm{~cm}^{-2}$. 
PS film shows oscillatory and noisy features and exhibits low values of capacitance $(\sim \mathrm{mF})$ showing the presence of defects/interface states [4].

Fig. 4(a-c) shows the diffraction curves recorded in symmetrical Bragg geometry for (400) diffracting planes for polished Si substrate before anodization, $\mathbf{E V}-\mathbf{A l}$ and SP-Al PS films $\left(\mathrm{I}_{\mathrm{d}} \sim 20 \mathrm{~mA} \mathrm{~cm}{ }^{-2}\right)$ respectively. Here, a sharp DC (Fig 4(a)) with full width at half maximum $(\mathrm{FWHM}) \sim 8$ arc $\mathrm{s}$ shows good quality $\mathrm{Si}$ substrate as this value is quite close to that expected from the plane wave dynamical theory of X-ray diffraction for a perfect crystal [5]. For EV-Al PS film, the DC shows only a single peak having the FWHM value of 11 arc s (Fig. 4(b)). It is well known that due to pores on the surface of Si crystal, the tensile stress develops due to expansion of the crystalline lattice surrounded by pores and dangling bonds and lead to a slight increase in the lattice constant of the PS film [4]. Due to change in lattice constant of PS film, another peak is expected at a lower diffraction angle with respective to the substrate peak. However, the observed single peak shows that either the PS film has low thickness $(\sim$ few $\mathrm{nm}$ as also observed from gravimetric results) due to which diffracted X-ray intensity could not be measured or the quality of the film is inferior. Similar features were observed for EV-Ag PS films as well. However, for SP-Al PS film, the DC (Fig. 4(c)) shows a well resolved satellite peak at an angular distance of -160 arc sec due to the porous film formed on the Si substrate. The inset in the Fig. 4(c) shows its amplified view. As confirmed from our earlier studies [3], the peak at lower angle with respect to the substrate peak position indicates that the perpendicular lattice constant of the PS film is more than that of the substrate. The strain $(\Delta \mathrm{a} / \mathrm{a})$ normal to the interface due to this lattice mismatch can be obtained from Bragg equation in its differential form given by,

$$
\Delta \mathrm{a} / \mathrm{a}=-\cot \theta_{\mathrm{B}} \Delta \theta
$$

where, $\Delta \theta$ is the angular separation between film and substrate peaks and $\theta_{\mathrm{B}}$ being the Bragg diffraction angle. The film peak at negative side shows that the film has a tensile stress due to pores around Si crystalline strands. For EV-Al PS film, (Fig. 4(b)) the strain obtained from Eqn. (1) is $(28.7 \pm 1) \times 10^{-4}$. Thus from above, the DC's shows that PS films formed using screen-printed contacts
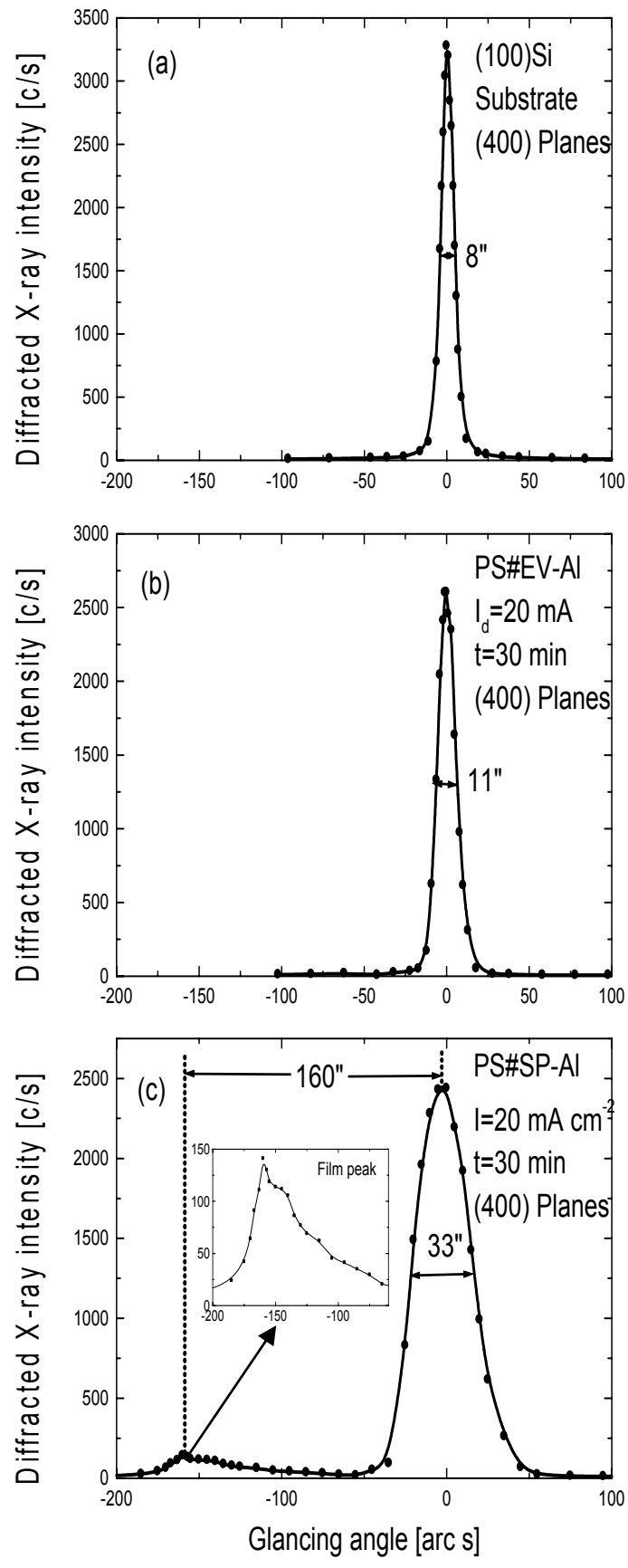

Fig. 4: Diffraction curves recorded for (400) diffracting planes using MoK $\alpha_{1}$ radiation: (a) Blank $S i$ wafer and (b) \& (c) $\boldsymbol{E} \boldsymbol{V}-\boldsymbol{A l}$ and $\boldsymbol{S P}-\boldsymbol{A l}$ PS films respectively. were superior in quality as compared to thermal evaporated ones. Fig. 5(a-c) shows DC of SP-Ag PS films corresponding to $\mathrm{I}_{\mathrm{d}} \sim 10,20 \& 30 \mathrm{~mA} \mathrm{~cm}$ respectively. For low $\mathrm{I}_{\mathrm{d}} \sim 10 \mathrm{~mA} \mathrm{~cm} \mathrm{c}^{-2}$, the DC (Fig. 5(a)) shows a satellite peak due to PS film at an angular distance of 138 arc s. Though the height of the film peak is much smaller than that of the substrate peak, the peak position could be measured with an uncertainty of \pm 2 arc s with an overall uncertainty of \pm 5 arc $\mathrm{s}$ in the value of angular distance between film and substrate peaks. The value of strain $(\Delta \mathrm{a} / \mathrm{a})$ thus obtained from the Eqn. (1) is $(24.7 \pm 1) \times 10^{-4}$. Similarly, for PS at $\mathrm{I}_{d}=20 \mathrm{~mA} \mathrm{~cm}{ }^{-2}$, the DC (Fig. 5(b)) shows a well resolved film peak with higher peak intensity as compared to fig. 5(a)) indicating increase in PS 
thickness with increase in $\mathrm{I}_{\mathrm{d}}$ from $10 \mathrm{~mA} \mathrm{~cm}^{-2}$ to $20 \mathrm{~mA} \mathrm{~cm}{ }^{-2}$ respectively. This is expected as the pore-depth increases with increase in $\mathrm{I}_{\mathrm{d}}$. However, the peak seperation also increases from 138 to 160 arc $\mathrm{s}$ with corresponding increase in tensile stress from its initial value of 24.7 to $28.7 \times 10^{-4}$. This may be attributed to the possibly a small increment of pore size in addition to increase in the pore depth. The increase in PL intensity \& its blue-shift with increase in $I_{d}$ from 10 to $20 \mathrm{~mA}$ $\mathrm{cm}^{-2}$ is also a testimonial to this. From fig. 4(c) $\& 5(\mathrm{~b})$, the peak separation is almost the same $(\sim$ 160 arc s) even though the back contacts are different (Ag instead of $\mathrm{Al}$ ). In contrast to the DCs of curves (a) and (b) of Fig. 5, curve (c) does not show any noticeable film peak as $I_{d}$ increases further to $30 \mathrm{~mA} \mathrm{~cm} \mathrm{~cm}^{-2}$. The disappearance of film peak at $\mathrm{I}_{\mathrm{d}} \sim 30 \mathrm{~mA} \mathrm{~cm} \mathrm{~cm}^{-2}$ (Fig. 4, (c)) indicates that at this $\mathrm{I}_{\mathrm{d}}$, the bonds between Si atoms of film and substrate no longer exists and the film peels off from the substrate as was also observed visually. Similar behavior is also observed for SP-AI PS films for $\mathrm{I}_{d}>20 \mathrm{~mA}$ $\mathrm{cm}^{-2}$.

\section{Conclusion}

High-resolution XRD, PL, SEM, FTIR \& capacitance-Voltage studies showed that the (SP-(Ag/AI)) PS films formed on polished Si (100) substrates under the same conditions of anodization are superior as compared to (EV(Ag/Al)) PS films respectively. PS layers with different back-contacts and their interfaces have been characterized by recording diffraction curves and measuring lattice mismatch/strain and the radius of curvature due to induced biaxial stress caused by the lattice expansion of PS film due to pores. SP-(Ag/Al) PS films exhibits better crystalline perfection, higher stability, higher film thickness, H-passivated surfaces and low defect density as compared to
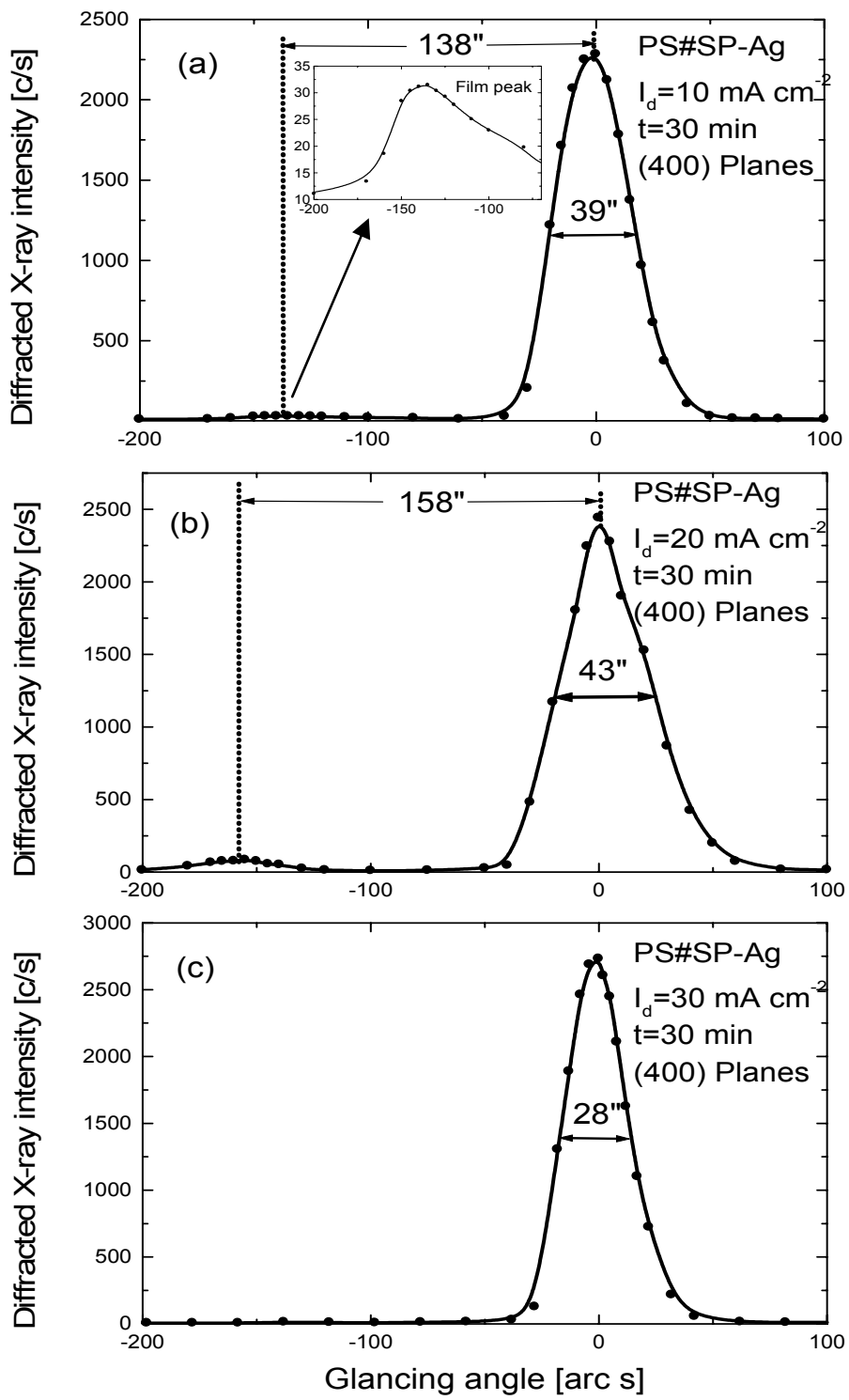

Fig. 5: Diffraction curves recorded for (400) diffracting planes using $M o K \alpha_{1}$ radiation for $\boldsymbol{S P}-\boldsymbol{A g}$ PS films at different $I_{d}:$ (a) 10, (b) 20 and (c) $30 \mathrm{~mA}$ $\mathrm{cm}^{-2}$.

EV-(Ag/Al) PS films. The results conclusively demonstrates the viability of screen -printing contact technology for the possible application of PS films in Si-solar cells.

\section{Acknowledgements}

We thank Director NPL for providing the facilities for the successful completion of this work. Thanks to Dr. Ritu Srivastava (NPL) for CV measurements. Priyanka acknowledges MNES for providing NRE fellowship. 


\section{References}

[1] T. Sun, J. Miao, R. Lin, Y. Fu, Sol. Energy Mater. Sol. Cells 85 (2005) 73.

[2] G. Cankaya, N. Ucar and A. Turut, Int. J. Electronics, 87 (10) (2000) 1171.

[3] G. Bhagavannarayana, Shailesh N. Sharma, R.K. Sharma And S.T. Lakshmikumar, Materials Chemistry and Physics, 97 (2-3), (2006) 442.

[4] Priyanka, Shailesh N Sharma, S. Salam, M. Husain and M. Lal, Sol. Energy Mats.\& Solar Cells, $91(15-16)(2007) 1510$.

[5] G. Bhagavannarayana and P. Zaumseil, J. Appl. Phys. 82, (1997) 1172. 